\title{
A Framework to Implement Organisational Performance Measurement in Health Charities
}

\author{
Richard Colbran ${ }^{1,}$, Robyn Ramsden ${ }^{2}$, Karen Stagnitti ${ }^{2}$, John Toumbourou ${ }^{3}$, Genevieve Pepin $^{2}$ \\ ${ }^{1}$ New South Wales (NSW) Rural Doctors Network, New South Wales, Australia \\ ${ }^{2}$ School of Health and Social Development, Deakin University, Victoria, Australia \\ ${ }^{3}$ School of Psychology, Deakin University, Victoria, Australia
}

Email address:

rcolbran@nswrdn.com.au (R. Colbran), rgwaghorne@gmail.com (R. Ramsden), karen.stagnitti@deakin.edu.au (K. Stagnitti), john.toumbourou@deakin.edu.au (J. Toumbourou), genevieve.pepin@deakin.edu.au (G. Pepin)

${ }^{*}$ Corresponding author

\section{To cite this article:}

Richard Colbran, Robyn Ramsden, Karen Stagnitti, John Toumbourou, Genevieve Pepin. A Framework to Implement Organisational Performance Measurement in Health Charities. Journal of Public Policy and Administration. Vol. 5, No. 1, 2021, pp. 13-23.

doi: $10.11648 /$ j.jppa.20210501.13

Received: February 25, 2021; Accepted: March 24, 2021; Published: March 30, 2021

\begin{abstract}
Organisational Performance Measurement (OPM) is a recognised management tool for business success, however it is under-reported and potentially under-utilised by non-acute health charities. This is the first study to recommend a set of methodological implementation factors for OPM in health charities that provide non-acute services. These factors consider the sector's unique governance, stakeholder and service delivery requirements. A cutting and sorting thematic analysis of data extracted from eligible sources of a PRISMA systematic literature review was used to generate a set of implementation factors and operating elements for organisational performance measurement (OPM) in non-acute health charities. These were then compared to OPM implementation factors for-profit, government and other not-for-profit. The study found 30 operating elements categorised into five implementation factors for successful OPM implementation for health charities: 1) OPM Implementation Plan (9 elements); 2) Commitment (5 elements); 3) Organisation Understanding and Learning (8 elements); 4$)$ Alignment, Integration and Resourcing (5 elements); and 5) Measures and Indicators (3 elements). These factors were packaged as the Framework for Non-Acute Health Charity Performance Implementation (NCPI Framework). The NCPI Framework may support the uptake of OPM within the sector. Case study evaluations of the NCPI Framework will now add value to its continued development.
\end{abstract}

Keywords: Performance, Organisation, Charity, Not-for-profit, Non-acute Health, Health

\section{Introduction}

Organisations, whether they are for-profit, government or not-for-profit, exist to allow groups of associated people accomplish common goals [1]. For success an organisation must reach sustainable competitive advantage whereby it attains and maintains the factors that contribute to outperforming competitors over long-periods [2, 3]. Organisational performance measurement (OPM) is the process of accounting and tracking an organisation's level of performance $[4,5]$. OPM has been demonstrated to be "essential to the survival and success of the modern business" [6]. OPM provides a foundation for governance by creating strategic clarity and coherence through evaluation, control, budgeting, motivating, promoting, celebrating, learning and improving [5]. There are many recognised OPM tools such as the Du Pont Model and Performance Prism however the Balanced Scorecard designed by Kaplan and Norton [7] has prevailed as the most widely accepted system [8].

OPM uptake within not-for-profit industry has been slow by comparison to for-profit and government industry [9]. This is despite increasing governance and service standard demands and a need to compete against other not-for-profits and private and government entities [10, 11]. Tailored implementation approaches that take into account nuances of industry, sector and individual organisations enhance the likelihood of OPM success and many researchers recommend 
that more should be done to understand factors that could encourage and support OPM uptake by not-for-profits [1215]. Others recognise that non-profit organisations are complex and multidimensional and require specialised understanding and support for OPM which responds to their uniqueness and nuances [16]. The lack of tailored OPM implementation strategies for non-profit sectors may be one of the reasons for poor uptake of OPM.

Non-acute health charities form part of the nongovernment health services and charities industry recognised by the World Health Organisation's Civil Society Initiative [17]. They are non-government, not-for-profit charitable organisations that provide non-hospital health services and their primary clinical purpose is non-hospital maintenance care which is defined as support for a patient with impairment or activity limitation due to a health condition and often requires care over an indefinite period following initial assessment or treatment as opposed to complex stabilisation in a complementary manner with other noncharity social services $[18-20]$. In the Australian context there are hundreds of non-acute and maintenance health providers with a combined annual turnover of $\$ 3$ billion through revenue streams such as government contracts, donations, fee-for-service and membership [21]. These include NSW Rural Doctors Network that provides tailored health workforce solutions for rural communities and Royal Far West that provides paediatric developmental support services. Organisations in this sector under-utilise or underreport OPM and an evidence-informed method for OPM implementation does not exist for the sector [18]. This brings into question the sector's capability to accurately measure and report organisational performance. To respond to the lack of a tailored OPM implementation approach for non-acute health charities, this study aimed to identify the important factors for OPM implementation for that sector.

\section{Method}

This study formed part of a research program investigating OPM in the non-acute health charity sector [18, 22]. Data suggesting barriers and success factors relating to OPM implementation in non-government organisations, charity organisations and not-for-profit healthcare organisations was extracted from non-fictional resource books and 20 peerreviewed journal articles. These data sources (shown in Table 1) were identified in the research group's earlier PRISMA systematic literature review [18]. That study found there was no published literature relating to OPM in non-acute health charities and that a methodologically developed OPM implementation model for the sector did not exist in published literature. As shown in Table 2, the sources were chosen as they reported OPM implementation factors from sectors that in the research group's opinion were most closely related to the non-acute health charity sector and were appraised to have a high level of trustworthiness using the Rosalind Franklin Qualitative Research Appraisal Instrument [23].

Through cutting and sorting thematic analysis informed by Ryan and Bernard [24] relevant statements or ideas in the literature were identified and clusters of similar OPM implementation data themes were created. These clusters were labelled as the key factors for implementation success and sub-themes were listed as operating elements enabling each factor. These factors and operating elements were then aggregated into a framework table.

Table 1. Data Sources for the study.

\begin{tabular}{|c|c|}
\hline Paper & Summary relating to OPM Implementation \\
\hline $\begin{array}{l}\text { "Measuring performance of non-profit organisations: } \\
\text { evidence from large charities" [16] }\end{array}$ & $\begin{array}{l}\text { Predominantly investigates the best measures of performance in charities. Using a hybrid } \\
\text { methodological approach investigated over } 100 \text { British charities from a range of sectors. Does not } \\
\text { specifically consider OPM implementation however provides detailed account of important } \\
\text { elements to performance measure development. }\end{array}$ \\
\hline "Strategic performance measurement in a healthcare & The study considers OPM implementation by GHD (Provisional Governmental Hospital of \\
\hline organisation: A multiple criteria approach based on & Didimoticho, Greece) which provides primary and hospital healthcare to a local region. \\
\hline balanced scorecard" [33] & Predominantly focuses on performance measures however provides implementation insights. \\
\hline $\begin{array}{l}\text { Assessing and Monitoring Strategy Implementation in } \\
\text { Health Care Organizations" [27] }\end{array}$ & The study reviews in detail the recommended use of OPM in healthcare organisations. \\
\hline $\begin{array}{l}\text { "The practice of the Balanced Scorecard in health } \\
\text { care" [30] }\end{array}$ & $\begin{array}{l}\text { Provides a detailed account of OPM theory and implementation in three Swedish health regions. } \\
\text { The paper highlights considerations for OPM usage in the health context. }\end{array}$ \\
\hline $\begin{array}{l}\text { "The establishment and comparison of the balanced } \\
\text { scorecard for profit and non-profit organizations" [53] }\end{array}$ & $\begin{array}{l}\text { Provides a review of literature and recommends important aspects of OPM development in non- } \\
\text { profit organisations. }\end{array}$ \\
\hline $\begin{array}{l}\text { Analysing BSC and IC's usefuleness in nonprofit } \\
\text { organisations [54] }\end{array}$ & $\begin{array}{l}\text { Provides a review of OPM and intellectual capital (IC) as strategic management methods in } \\
\text { nonprofit organisations }\end{array}$ \\
\hline $\begin{array}{l}\text { "Using a Balanced Scorecard in a Nonprofit } \\
\text { Organization" [15] }\end{array}$ & $\begin{array}{l}\text { Provides information for adapting the OPM for implementation in Non-profit organisations. Key } \\
\text { elements include common mistakes, creating organisational measures and categories and finally } \\
\text { seven steps for implementation. }\end{array}$ \\
\hline $\begin{array}{l}\text { "The Balanced Scorecard of acute settings: } \\
\text { development process, definition of } 20 \text { strategic } \\
\text { objectives and implementation" [35] }\end{array}$ & $\begin{array}{l}\text { Study presents the steps and lessons learned during a 5-year project that aimed at the organisation- } \\
\text { wide implementation of the Health Promoting Hospital Strategy using the Balanced Scorecard. }\end{array}$ \\
\hline
\end{tabular}




\begin{tabular}{|c|c|}
\hline Paper & Summary relating to OPM Implementation \\
\hline $\begin{array}{l}\text { "Applying the balanced scorecard to local public } \\
\text { health performance measurement: deliberations and } \\
\text { decisions" [55] }\end{array}$ & $\begin{array}{l}\text { Study details a two-year project introducing a public-health specific balanced scorecard } \\
\text { framework in Canada. }\end{array}$ \\
\hline $\begin{array}{l}\text { "Implementing a balanced scorecard as a strategic } \\
\text { management tool in a long-term care organization" } \\
{[14]}\end{array}$ & $\begin{array}{l}\text { The study details a Canadian health organisations implementation of the OPM and provides } \\
\text { insights and lessons learnt. }\end{array}$ \\
\hline $\begin{array}{l}\text { "Implementing A Balanced Scorecard In A Not-For- } \\
\text { Profit Organization" [34] }\end{array}$ & $\begin{array}{l}\text { Examines the use of the OPM in a not-for-profit health service. Provides an account of the } \\
\text { application of the OPM and provides discussion on its implications and recommends important } \\
\text { aspects for its use. }\end{array}$ \\
\hline $\begin{array}{l}\text { "Lives in the balance: an analysis of the balanced } \\
\text { scorecard (BSC) in healthcare organizations" [56] }\end{array}$ & $\begin{array}{l}\text { A review of published literature citing } 22 \text { examples of OPM in health care organizations. Study } \\
\text { referred to hospital systems, hospitals and hospital departments, a psychiatric centre, National } \\
\text { health-care systems and local government. Provides detailed account of appropriate indicators of } \\
\text { health organisation performance and considers important implementation factors. }\end{array}$ \\
\hline $\begin{array}{l}\text { "An integrated Balanced Scorecard strategic planning } \\
\text { model for nonprofit organisations" [57] }\end{array}$ & $\begin{array}{l}\text { Describes an implementation model for nonprofit organisations and proposes a practical example } \\
\text { within a non-health not-for-profit. }\end{array}$ \\
\hline $\begin{array}{l}\text { "The Application of Niven's Balanced Scorecard in a } \\
\text { Not-For-Profit Organization in Hong Kong: What are } \\
\text { the Factors for Success" [58] }\end{array}$ & $\begin{array}{l}\text { The study examines the extent to which a OPM model could be applied to a non-profit } \\
\text { organisation. }\end{array}$ \\
\hline $\begin{array}{l}\text { "A study of implementing Balanced Scorecard (BSC) } \\
\text { in non-profit organizations: A case study of private } \\
\text { hospital" }[59]\end{array}$ & $\begin{array}{l}\text { Provides information for } 17 \text { steps for OPM implementation and then provides an example of how } \\
\text { each step could be translated into a sample organisation. }\end{array}$ \\
\hline "Charity's changed environment" [60] & $\begin{array}{l}\text { The study reviews the need to evaluate the impact and effectiveness of volunteer (charitable) } \\
\text { organizations in Canada. Considers OPM however predominantly investigates ISO Quality } \\
\text { Management System to benchmark and improve performance. Provides implementation insights in } \\
\text { the charitable sector which are useful. }\end{array}$ \\
\hline $\begin{array}{l}\text { Defining, justisfying and implementing the Balanced } \\
\text { Scorecard in the National Health Service" [29] }\end{array}$ & $\begin{array}{l}\text { The study offers insights into OPM implementation progress made by a Primary Care Trust (UK) } \\
\text { within the Bradford Health Action Zone (UK). } \\
\text { The study considers OPM implementation in an NHS multi-agency setting (Bradford Health }\end{array}$ \\
\hline $\begin{array}{l}\text { "Success factors for implementation of the balanced } \\
\text { scorecard in a HNS multi-agency setting" [61] }\end{array}$ & $\begin{array}{l}\text { Action Zone, UK). Demonstrates background for supporting OPM usage, highlights conceptual } \\
\text { concerns and outlines factors for successful OPM implementation. Argues that if key criteria are } \\
\text { met successful OPM implementation is possible. }\end{array}$ \\
\hline $\begin{array}{l}\text { "Applying the Balanced Scorecard in Healthcare } \\
\text { Provider Organizations" [31] }\end{array}$ & $\begin{array}{l}\text { One of the originally published papers regarding OPM in healthcare. Reviews OPM experiences } \\
\text { of nine healthcare organizations categorsied as Integrated Delivery Systems, Academic Medical } \\
\text { Center, Skilled Nursing Facility or Community Hospital. Considers organizational motivation, } \\
\text { implementation factors and challenges and barriers encountered. Finally suggests five guidelines } \\
\text { for successful implementation are offered. }\end{array}$ \\
\hline
\end{tabular}

Table 2. Data Source Suitability for the study.

\begin{tabular}{|c|c|c|c|c|c|c|c|}
\hline \multirow[b]{2}{*}{ Paper } & \multirow{2}{*}{$\begin{array}{l}\text { Evidence-level Assessment } \\
\text { Rosalind Franklin } \\
\text { Qualitative Research } \\
\text { Appraisal Instrument (RF- } \\
\text { QRA) }\end{array}$} & \multicolumn{5}{|c|}{ Organisation type/s analysed for OPM implementation } & \multirow[b]{2}{*}{$\begin{array}{l}\text { Theoretical or } \\
\text { Organisational } \\
\text { Case Study }\end{array}$} \\
\hline & & $\begin{array}{l}\text { Non- } \\
\text { Government }\end{array}$ & $\begin{array}{l}\text { Non- } \\
\text { Profit }\end{array}$ & Charity & $\begin{array}{l}\text { Non-Acute } \\
\text { Health } \\
\text { Service } \\
\text { Provision } \\
\end{array}$ & $\begin{array}{l}\text { Other Primary } \\
\text { Health Service } \\
\text { Provision }\end{array}$ & \\
\hline $\begin{array}{l}\text { "Measuring performance of non- } \\
\text { profit organisations: evidence from } \\
\text { large charities" [16] }\end{array}$ & $\begin{array}{l}\text { RF-QRA - Level } 2 \\
\text { Credibility: Sound } \\
\text { Transferability: Sound } \\
\text { Dependability: Sound } \\
\text { Confirmability: Strong }\end{array}$ & $\mathrm{X}$ & $\mathrm{X}$ & $\mathrm{X}$ & & & Theoretical \\
\hline $\begin{array}{l}\text { "Strategic performance measurement } \\
\text { in a healthcare organisation: A } \\
\text { multiple criteria approach based on } \\
\text { balanced scorecard" [33] }\end{array}$ & $\begin{array}{l}\text { RF-QRA - Level } 2 \\
\text { Credibility: Strong } \\
\text { Transferability: Sound } \\
\text { Dependability: Sound } \\
\text { Confirmability: Sound }\end{array}$ & & & Possible & & $\begin{array}{l}\text { X } \\
\text { (Mixed care } \\
\text { services. } \\
\text { Primarily } \\
\text { acute) }\end{array}$ & $\begin{array}{l}\text { Organisational } \\
\text { Case Study }\end{array}$ \\
\hline $\begin{array}{l}\text { "The Balanced Scorecard as a } \\
\text { Management Tool for Assessing and } \\
\text { Monitoring Strategy Implementation } \\
\text { in Health Care Organizations" [27] }\end{array}$ & $\begin{array}{l}\text { RF-QRA - Level } 2 \\
\text { Credibility: Strong } \\
\text { Transferability: Sound } \\
\text { Dependability: Sound } \\
\text { Confirmability: Sound }\end{array}$ & & & & & $\begin{array}{l}X \\
\text { (Mixed service } \\
\text { types. Not } \\
\text { specifically } \\
\text { listed) }\end{array}$ & Theoretical \\
\hline $\begin{array}{l}\text { "The practice of the Balanced } \\
\text { Scorecard in health care" [30] }\end{array}$ & $\begin{array}{l}\text { RF-QRA - Level } 2 \\
\text { Credibility: Strong } \\
\text { Transferability: Sound } \\
\text { Dependability: Sound } \\
\text { Confirmability: Sound }\end{array}$ & & & & & $\begin{array}{l}\text { X } \\
\text { (Region health } \\
\text { services. } \\
\text { Primarily } \\
\text { hospital } \\
\text { focused) }\end{array}$ & $\begin{array}{l}\text { Organisational } \\
\text { Case Study }\end{array}$ \\
\hline $\begin{array}{l}\text { "The establishment and comparison } \\
\text { of the balanced scorecard for profit }\end{array}$ & $\begin{array}{l}\text { RF-QRA - Level } 2 \\
\text { Credibility: Sound }\end{array}$ & $\mathrm{X}$ & $\mathrm{X}$ & & & & Theoretical \\
\hline
\end{tabular}




\begin{tabular}{|c|c|c|c|c|c|c|c|}
\hline \multirow[b]{2}{*}{ Paper } & \multirow{2}{*}{$\begin{array}{l}\text { Evidence-level Assessment } \\
\text { Rosalind Franklin } \\
\text { Qualitative Research } \\
\text { Appraisal Instrument (RF- } \\
\text { QRA) } \\
\end{array}$} & \multicolumn{5}{|c|}{ Organisation type/s analysed for OPM implementation } & \multirow[b]{2}{*}{$\begin{array}{l}\text { Theoretical or } \\
\text { Organisational } \\
\text { Case Study }\end{array}$} \\
\hline & & $\begin{array}{l}\text { Non- } \\
\text { Government }\end{array}$ & $\begin{array}{l}\text { Non- } \\
\text { Profit }\end{array}$ & Charity & $\begin{array}{l}\text { Non-Acute } \\
\text { Health } \\
\text { Service } \\
\text { Provision } \\
\end{array}$ & $\begin{array}{l}\text { Other Primary } \\
\text { Health Service } \\
\text { Provision }\end{array}$ & \\
\hline and non-profit organizations" & Transferability: Sound & & & & & & \\
\hline [53] & $\begin{array}{l}\text { Dependability: Sound } \\
\text { Confirmability: Sound } \\
\text { RF-QRA - Level } 2\end{array}$ & & & & & & \\
\hline $\begin{array}{l}\text { Analysing BSC and IC's usefuleness } \\
\text { in nonprofit organisations [54] }\end{array}$ & $\begin{array}{l}\text { Credibility: Strong } \\
\text { Transferability: Sound } \\
\text { Dependability: Strong } \\
\text { Confirmability: Sound }\end{array}$ & $\mathrm{X}$ & $X$ & & & & Theoretical \\
\hline $\begin{array}{l}\text { "Implementing Balanced Scorecard } \\
\text { for Performance Measurement" [28] }\end{array}$ & $\begin{array}{l}\text { RF-QRA - Level } 2 \\
\text { Credibility: Sound } \\
\text { Transferability: Sound } \\
\text { Dependability: Sound } \\
\text { Confirmability: Sound } \\
\text { RF-QRA - Level } 2\end{array}$ & & & & & & Theoretical \\
\hline $\begin{array}{l}\text { "Using a Balanced Scorecard in a } \\
\text { Nonprofit Organization" [15] }\end{array}$ & $\begin{array}{l}\text { Credibility: Sound } \\
\text { Transferability: Sound } \\
\text { Dependability: Sound } \\
\text { Confirmability: Sound }\end{array}$ & $\mathrm{X}$ & $\mathrm{X}$ & & & & Theoretical \\
\hline $\begin{array}{l}\text { "The Balanced Scorecard of acute } \\
\text { settings: development process, } \\
\text { definition of } 20 \text { strategic objectives } \\
\text { and implementation" [35] }\end{array}$ & $\begin{array}{l}\text { RF-QRA - Level } 2 \\
\text { Credibility: Sound } \\
\text { Transferability: Sound } \\
\text { Dependability: Sound } \\
\text { Confirmability: Sound }\end{array}$ & & & & & $\begin{array}{l}\text { X } \\
\text { (Mixed Care. } \\
\text { Primarily } \\
\text { Hospitals) }\end{array}$ & $\begin{array}{l}\text { Organisational } \\
\text { Case Study }\end{array}$ \\
\hline $\begin{array}{l}\text { "Applying the balanced scorecard to } \\
\text { local public health performance } \\
\text { measurement: deliberations and } \\
\text { decisions" [55] }\end{array}$ & $\begin{array}{l}\text { RF-QRA - Level } 2 \\
\text { Credibility: Sound } \\
\text { Transferability: Sound } \\
\text { Dependability: Sound } \\
\text { Confirmability: Sound }\end{array}$ & & & & & $\begin{array}{l}\text { X } \\
\text { (Mixed care } \\
\text { services. } \\
\text { Primarily } \\
\text { public health) }\end{array}$ & $\begin{array}{l}\text { Organisational } \\
\text { Case Stud }\end{array}$ \\
\hline $\begin{array}{l}\text { "Implementing a balanced scorecard } \\
\text { as a strategic management tool in a } \\
\text { long-term care organization" [14] }\end{array}$ & $\begin{array}{l}\text { RF-QRA - Level } 2 \\
\text { Credibility: Strong } \\
\text { Transferability: Strong } \\
\text { Dependability: Sound } \\
\text { Confirmability: Sound }\end{array}$ & $\mathrm{X}$ & $X$ & Possible & & $\begin{array}{l}\text { X } \\
\text { (Mixed care. } \\
\text { Primarily aged } \\
\text { care) }\end{array}$ & $\begin{array}{l}\text { Organisational } \\
\text { Case Study }\end{array}$ \\
\hline $\begin{array}{l}\text { "Implementing A Balanced } \\
\text { Scorecard In A Not-For-Profit } \\
\text { Organization" [34] }\end{array}$ & $\begin{array}{l}\text { RF-QRA - Level } 2 \\
\text { Credibility: Sound } \\
\text { Transferability: Strong } \\
\text { Dependability: Sound } \\
\text { Confirmability: Sound } \\
\text { RF-QRA - Level } 2\end{array}$ & $\mathrm{X}$ & $\mathrm{X}$ & & & $\begin{array}{l}\text { X } \\
\text { (Rehabilitation } \\
\text { Centre) }\end{array}$ & $\begin{array}{l}\text { Organisational } \\
\text { Case Study }\end{array}$ \\
\hline $\begin{array}{l}\text { "Lives in the balance: an analysis of } \\
\text { the balanced scorecard (BSC) in } \\
\text { healthcare organizations" [56] }\end{array}$ & $\begin{array}{l}\text { Credibility: Sound } \\
\text { Transferability: Strong } \\
\text { Dependability: Sound } \\
\text { Confirmability: Sound } \\
\text { RF-QRA - Level } 2\end{array}$ & & & & & $\begin{array}{l}X \\
\text { (Mixed service } \\
\text { types) }\end{array}$ & Theoretical \\
\hline $\begin{array}{l}\text { "An integrated Balanced Scorecard } \\
\text { strategic planning model for } \\
\text { nonprofit organisations" [57] }\end{array}$ & $\begin{array}{l}\text { Credibility: Sound } \\
\text { Transferability: Sound } \\
\text { Dependability: Sound } \\
\text { Confirmability: Sound }\end{array}$ & $\mathrm{X}$ & $\mathrm{X}$ & & & & Theoretical \\
\hline $\begin{array}{l}\text { "The Application of Niven's } \\
\text { Balanced Scorecard in a Not-For- } \\
\text { Profit Organization in Hong Kong: } \\
\text { What are the Factors for Success" } \\
\text { [58] }\end{array}$ & $\begin{array}{l}\text { RF-QRA - Level } 2 \\
\text { Credibility: Sound } \\
\text { Transferability: Strong } \\
\text { Dependability: Sound } \\
\text { Confirmability: Sound }\end{array}$ & $\mathrm{X}$ & $\mathrm{X}$ & & $\mathrm{X}$ & $\begin{array}{l}\text { Social welfare } \\
\text { organisation } \\
\text { providing day- } \\
\text { care nursing } \\
\text { and } \\
\text { rehabilitation } \\
\text { services }\end{array}$ & $\begin{array}{l}\text { Organisational } \\
\text { Case Study }\end{array}$ \\
\hline $\begin{array}{l}\text { "A study of implementing Balanced } \\
\text { Scorecard (BSC) in non-profit } \\
\text { organizations: A case study of } \\
\text { private hospital" [59] } \\
\text { "Charity's changed environment" } \\
\text { [60] }\end{array}$ & $\begin{array}{l}\text { RF-QRA - Level } 2 \\
\text { Credibility: Sound } \\
\text { Transferability: Strong } \\
\text { Dependability: Sound } \\
\text { Confirmability: Sound } \\
\text { RF-QRA - Level } 2 \\
\text { Credibility: Sound } \\
\text { Transferability: Sound }\end{array}$ & $\mathrm{X}$ & $\mathrm{X}$ & $\mathrm{X}$ & & $\begin{array}{l}\text { X } \\
\text { (Mixed care } \\
\text { services. } \\
\text { Primarily } \\
\text { acute) }\end{array}$ & $\begin{array}{l}\text { Organisational } \\
\text { Case Study }\end{array}$ \\
\hline
\end{tabular}




\begin{tabular}{|c|c|c|c|c|c|c|c|}
\hline \multirow[b]{2}{*}{ Paper } & \multirow{2}{*}{$\begin{array}{l}\text { Evidence-level Assessment } \\
\text { Rosalind Franklin } \\
\text { Qualitative Research } \\
\text { Appraisal Instrument (RF- } \\
\text { QRA) }\end{array}$} & \multicolumn{5}{|c|}{ Organisation type/s analysed for OPM implementation } & \multirow[b]{2}{*}{$\begin{array}{l}\text { Theoretical or } \\
\text { Organisational } \\
\text { Case Study }\end{array}$} \\
\hline & & $\begin{array}{l}\text { Non- } \\
\text { Government }\end{array}$ & $\begin{array}{l}\text { Non- } \\
\text { Profit }\end{array}$ & Charity & $\begin{array}{l}\text { Non-Acute } \\
\text { Health } \\
\text { Service } \\
\text { Provision } \\
\end{array}$ & $\begin{array}{l}\text { Other Primary } \\
\text { Health Service } \\
\text { Provision }\end{array}$ & \\
\hline $\begin{array}{l}\text { Defining, justisfying and } \\
\text { implementing the Balanced } \\
\text { Scorecard in the National Health } \\
\text { Service" [29] }\end{array}$ & $\begin{array}{l}\text { Dependability: Sound } \\
\text { Confirmability: Sound } \\
\text { RF-QRA - Level } 2 \\
\text { Credibility: Strong } \\
\text { Transferability: Sound } \\
\text { Dependability: Strong } \\
\text { Confirmability: Sound }\end{array}$ & $\mathrm{X}$ & $\mathrm{X}$ & & & $\begin{array}{l}\text { X } \\
\text { (Mixed care } \\
\text { services. } \\
\text { Primarily } \\
\text { acute) }\end{array}$ & $\begin{array}{l}\text { Organisational } \\
\text { Case Study }\end{array}$ \\
\hline $\begin{array}{l}\text { "Success factors for implementation } \\
\text { of the balanced scorecard in a HNS } \\
\text { multi-agency setting" [61] }\end{array}$ & $\begin{array}{l}\text { RF-QRA - Level } 1 \\
\text { Credibility: Strong } \\
\text { Transferability: Sound } \\
\text { Dependability: Strong } \\
\text { Confirmability: Strong }\end{array}$ & $\mathrm{X}$ & $\mathrm{X}$ & & & $\begin{array}{l}\text { X } \\
\text { (Mixed care } \\
\text { services. } \\
\text { Primarily } \\
\text { acute) }\end{array}$ & $\begin{array}{l}\text { Organisational } \\
\text { Case Study }\end{array}$ \\
\hline $\begin{array}{l}\text { "Applying the Balanced Scorecard in } \\
\text { Healthcare Provider Organizations" } \\
\text { [31] }\end{array}$ & $\begin{array}{l}\text { RF-QRA - Level } 2 \\
\text { Credibility: Strong } \\
\text { Transferability: Sound } \\
\text { Dependability: Strong } \\
\text { Confirmability: Sound }\end{array}$ & & & & & $\begin{array}{l}X \\
\text { (Mixed service } \\
\text { types. Not } \\
\text { specifically } \\
\text { listed) }\end{array}$ & $\begin{array}{l}\text { Organisational } \\
\text { Case Study }\end{array}$ \\
\hline
\end{tabular}

Finally, an analysis of these implementation factors was conducted comparing OPM implementation factors across different industries recommended in literature published between 2016 to 2019 .

\section{Results}

The thematic analysis of data produced a list of five factors for OPM implementation which incorporate 30 operational elements. Table 3 presents these factors and operating elements which the authors have titled as the Framework for
Non-Acute Health Charity Performance Implementation (NCPI Framework). The breakdown of the factors and operating elements are:

1. Factor 1: OPM Implementation Plan (9 elements)

2. Factor 2: Commitment (5 elements)

3. Factor 3: Understanding and Learning (8 elements)

4. Factor 4: Alignment, Integration and Resourcing (5 elements)

5. Factor 5: Measures and Indicators (3 elements)

The following evidence supports the five implementation factors.

Table 3. A Framework for Non-Acute Health Charity Performance Implementation (NCPI Framework).

\begin{tabular}{|c|c|}
\hline OPM Implementation Factor and Operating Elements & Study Data Reference \\
\hline \multicolumn{2}{|l|}{ Factor 1: OPM Implementation Plan } \\
\hline $\begin{array}{l}\text { Element 1.1: Development and endorsement of a formal OPM implementation plan which } \\
\text { utilises evidence }\end{array}$ & $\begin{array}{l}{[35 ; 31, \text { p. } 18,187 \text { and } 93 ; 14, \text { p. } 8 ; 28, \text { p. } 12 ; 61, \text { p. } 106 ;} \\
59, \text { p. } 291]\end{array}$ \\
\hline Element 1.2: Translate organisational vision and strategy into tangible objectives and measures & $\begin{array}{l}{[31, \text { p. } 181 ; 29, \text { p. } 179 \text { and } 180 ; 57, \text { p. } 32 ; 28, \text { p. } 11 ; 59 \text {, }} \\
\text { p. 290] }\end{array}$ \\
\hline Element 1.3: Existence of a formal organisation strategy & {$[27$, p. $921 ; 14$, p. $9 ; 28$, p. $12 ; 15$, p. 10$]$} \\
\hline $\begin{array}{l}\text { Element 1.4: Articulation of organisational strategy, OPM Implementation Plan and cause-and- } \\
\text { effect relationships in a Strategy Map }\end{array}$ & $\begin{array}{l}{[27, \text { p. } 922 ; 35, \text { p. } 261 ; 54, \text { p. } 288 ; 57, \text { p. } 30 ; 28, \text { p. } 12 ;} \\
59, \text { p. } 295 ; 58, \text { p. } 32]\end{array}$ \\
\hline $\begin{array}{l}\text { Element 1.5: Go beyond short-term agendas and pay attention to medium and long-term } \\
\text { objectives }\end{array}$ & {$[29$, p. $177 ; 14$, p. 8 and 9$]$} \\
\hline Element 1.6: Confirm who is responsible for which actions and activities & {$[30$, p. 431$]$} \\
\hline Element 1.7: Ensure OPM model is adapted to meet unique organisational realities and demands & $\begin{array}{l}{[27, \text { p. } 922 \text { and } 924 ; 53, \text { p. } 3008 ; 33, \text { p. } 105 ; 56, \text { p. } 18)} \\
{[30, \text { p. } 431 ; 29, \text { p. } 185 ; 14, \text { p. } 9 \text { and } 13 ; 15, \text { p. } 10]}\end{array}$ \\
\hline Element 1.8: Acknowledge and plan for OPM deployment over extended time period & $\begin{array}{l}{[31, \text { p. } 185 ; 29, \text { p. } 185 ; 57, \text { p. } 28 ; 14, \text { p. } 8,9 \text { and } 13]} \\
\text { (Weir et al., 2009, p. } 6)[15, \text { p. } 10)\end{array}$ \\
\hline \multicolumn{2}{|l|}{ Factor 2: Commitment } \\
\hline Element 2.1: Demonstrated and continued Executive and Senior Management support for OPM & $\begin{array}{l}{[27, \text { p. } 924 ; 35, \text { p. } 268 ; 31, \text { p. } 181,185 \text { and } 186 ; 30, \text { p. }} \\
431 ; 14, \text { p. } 8,9 \text { and } 13 ; 55 \text {, p. } 6 ; 59, \text { p. } 291]\end{array}$ \\
\hline Element 2.2: Secure full organisational support for OPM implementation & $\begin{array}{l}{[31, \text { p. } 185 \text { and } 187 ; 30, \text { p. } 431 ; 34, \text { p. } 73 ; 29, \text { p. } 187 ; 61,} \\
\text { p. } 103 \text { and } 105]\end{array}$ \\
\hline Element 2.3: Organisational culture is appropriate and receptive to OPM implementation & {$[31$, p. $192 ; 14$, p. 8 and 13$]$} \\
\hline $\begin{array}{l}\text { Element 2.4: Creation of a OPM Implementation Steering Committee - potentially with mixed } \\
\text { representation across staff }\end{array}$ & {$[33$, p. $117 ; 60$, p. $27 ; 14$, p. $13 ; 59$, p. 291$]$} \\
\hline $\begin{array}{l}\text { Element 2.5: Appointment of a OPM Implementation Coordinator as a sole role or within } \\
\text { existing role }\end{array}$ & {$[30$, p. $442 ; 57$, p. $33 ; 14$, p. $13 ; 15$, p. 12$]$} \\
\hline
\end{tabular}




\begin{tabular}{|c|c|}
\hline OPM Implementation Factor and Operating Elements & Study Data Reference \\
\hline Element 3.1: Participation of staff in OPM design and revisions & $\begin{array}{l}{[53, \text { p. } 3010 ; 33 \text {, p. } 117 ; 35, \text { p. } 264 ; 31, \text { p. } 181 ; 30, \text { p. }} \\
442 ; 60, \text { p. } 27 ; 34 \text {, p. } 73 ; 61 \text {, p. } 105 ; 59 \text {, p. } 293]\end{array}$ \\
\hline $\begin{array}{l}\text { Element 3.2: Communicate organisational strategy, OPM purpose, the Implementation Plan and } \\
\text { its status and ensure staff are aware OPM is for strategic management not just performance } \\
\text { measurement }\end{array}$ & $\begin{array}{l}{[35, \text { p. } 268 ; 33 \text {, p. } 117 ; 30 \text {, p. } 430 \text { and } 431 ; 29, \text { p. } 180} \\
14 \text {, p. } 8,9,12 \text { and } 13 ; 59 \text {, p. } 291 ; 58, \text { p. } 31]\end{array}$ \\
\hline Element 3.3: Address any conceptual barriers to OPM within the organisation & {$[35$, p. $269 ; 29$, p. $185 ; 61$, p. 102$]$} \\
\hline $\begin{array}{l}\text { Element 3.4: Provide feedback to learn about and improve organisational strategy and OPM } \\
\text { Implementation Plan }\end{array}$ & $\begin{array}{l}{[35 \text {, p. } 268 ; 31, \text { p. } 187 \text { and } 193 ; 29, \text { p. } 180 ; 59, \text { p. } 292 ;} \\
15, \text { p. } 10 \text { and } 12]\end{array}$ \\
\hline Element 3.5: Facilitate periodic and systematic OPM review, adjustment and improvement & {$[33$, p. $117 ; 31$, p. $181 ; 29$, p. $180 ; 14$, p. 13$]$} \\
\hline Element 3.6: Identify and support OPM champions across the organisation & {$[30$, p. 439 and $442 ; 14$, p. 8$]$} \\
\hline $\begin{array}{l}\text { Element 3.7: Skills and tools in data analysis and management, and implementing feedback and } \\
\text { learning systems in-place }\end{array}$ & $\begin{array}{l}{[33, \text { p. } 117 ; 31, \text { p. } 187 ; 54, \text { p. } 291 ; 14, \text { p. } 13 ; 28, \text { p. } 12 ;} \\
58, \text { p. } 31 ; 15 \text {, p. } 10]\end{array}$ \\
\hline $\begin{array}{l}\text { Element } 3.8 \text { : Use team-based collaborative approaches among disciplines that do not regularly } \\
\text { work together }\end{array}$ & {$[33$, p. $117 ; 35$, p. $268 ; 31$, p. 188 and 193$]$} \\
\hline Factor 4: Alignment \& Integration (inc. Resourcing) & \\
\hline Element 4.1: Identify and align strategic initiatives & {$[29$, p. $180 ; 14$, p. 13$)$} \\
\hline $\begin{array}{l}\text { Element 4.2: Integrate OPM within existing management processes, governance mechanisms, } \\
\text { policies and reporting systems }\end{array}$ & {$[14$, p. 8,9 and $13 ; 15$, p. 12$]$} \\
\hline $\begin{array}{l}\text { Element 4.3: Designate OPM implementation within the organisation's Business and } \\
\text { Operational Plans }\end{array}$ & {$[29$, p. $185 ; 14$, p. 8 and 9$]$} \\
\hline Element 4.4: Acknowledge and prepare for OPM deployment resourcing investment & $\begin{array}{l}{[53, \text { p. } 3011 ; 35 \text {, p. } 269 ; 31, \text { p. } 185 \text { and } 193 ; 29, \text { p. } 185} \\
\text { and } 187 ; 14 \text {,p. } 8 ; 59 \text {, p. } 295 ; 15 \text {, p. } 12]\end{array}$ \\
\hline $\begin{array}{l}\text { Element 4.5: Cascading OPM accountability in departmental and personal goals throughout } \\
\text { organisation } \\
\text { Factor 5: Measures \& Indicators }\end{array}$ & $\begin{array}{l}{[27, \text { p. } 924 ; 33, \text { p. } 117 ; 31, \text { p. } 185 \text { and } 194 ; 57, \text { p. } 34 ; 14 \text {, }} \\
\text { p. } 13 ; 28, \text { p. } 12 ; 59, \text { p. } 295]\end{array}$ \\
\hline $\begin{array}{l}\text { Element 5.1: Measures and indicators customised to the organisation to represent all dimensions } \\
\text { of the organisation }\end{array}$ & $\begin{array}{l}{[27, \text { p. } 922 ; 16, \text { p. } 69 ; 33, \text { p. } 106 ; 56, \text { p. } 7 \text { and } 9)[31, \text { p. }} \\
186 ; 30 \text {, p. } 430 \text { and } 431 ; 34, \text { p. } 73 ; 14 \text {, p. } 8,9 \text { and } 13] \\
{[28, \text { p. } 10 \text { and } 12 ; 55, \text { p. } 6 ; 15 \text {, p. } 11 \text { and } 12)}\end{array}$ \\
\hline Element 5.2: Ensure targets are set for each measure and measures and indicators are meaningful & {$[28$, p. 10 and $12 ; 59$, p. $295 ; 15$, p. 10$)$} \\
\hline $\begin{array}{l}\text { Element 5.3: Ensure shared vision amongst staff cohort (esp. clinicians and managers) regarding } \\
\text { priority measures and indicators }\end{array}$ & {$[27$, p. $926 ; 55$, p. 6$]$} \\
\hline
\end{tabular}

\subsection{Factor 1: Implementation Planning}

OPM implementation is difficult and if not well planned will "be a disappointing waste of time" [15]. Niven [25] and Nair [27], along with six of the journal records, highlight the importance of implementation planning. Nine operating elements are recommended including: a formal OPM Implementation Plan (Element 1.1) to roadmap activities and encourage a project mindset [26]. The plan should consider unique structures, culture, capabilities and biases to enable the development, endorsement, activation and tracking of an organisational-wide systems approach to deployment [26, 25]. The plan should also include tangible project objectives and measures (Element 1.2). These first elements should be supported by an explicit organisational strategy (Element 1.3) so that the organisation's vision can be translated into operational terms [27].

An easy to understand Strategy Map (Element 1.4) should support the plan. The map should identify and illustrate the cause-effect relationships between strategy and implementation objectives [27, 28]. The plan must also pay attention to medium and long-term strategic objectives (Element 1.5) rather than short-term agendas [29] and confirm who is responsible for actions and activities (Element 1.6) [30]. Ensuring customisation of the approach to fit the organisation (Element 1.7) is critical as OPM implementation is not like following a recipe [14] and it should not be "copied blindly under the assumption that one size fits all" [15].
Finally, it is important to recognise that OPM deployment will take an extended time period (Element 1.8). Inamdar and Kaplan [31] suggest OPM implementation can take up to five years. Zimmerman [15] also recommended pre-planning implementation processes should not be rushed and consider the resource investment and organisational-wide implications of OPM implementation. Implementation of test experiences prior to full-scale execution to improve implementation understanding and processes [15] was also recommended through the literature (Element 1.9).

\subsection{Factor 2: Commitment}

Successful OPM implementation requires an absolute long-term whole-of-organisation commitment. This factor was strongly recognised by Nair [26], Smith [32] and Niven [25], along with 14 of the 20 journal records (see Table 3).

Considering implementation may take years, sustained executive and management commitment (Element 2.1) underpins success or failure as a lack of support is the number-one reason for OPM project failure [26]. Management must drive OPM introduction [14], encourage linkage of organisational strategy [27] and mobilise change across the organisation [31]. Radnor and Lovell [29] suggest that doubts regarding the concept of OPM inhibit its usage and that the ability to secure support from all the people in the organisation (Element 2.2), including clinicians who are not traditionally involved in business strategy, is critical for implementation success [30]. 
Organisational readiness and culture (Element 2.3) contributes to securing organisational commitment, as success requires a culture of receptiveness and learning [14, 31]. The creation of an Implementation Steering Committee (Element 2.4) and support for evidence-informed decisionmaking culture were cited as elements that support implementation and demonstrate commitment [33]. Dedicated staff positions to lead OPM implementation (Element 2.5) were also cited as success contributors [30].

\subsection{Factor 3: Organisation Understanding \& Learning}

Staff awareness and understanding of implementation were identified in 15 of the 20 journal papers (see Table 3 ). Niven [25] highlights five staff engagement criteria - contact; awareness; conceptual understanding; tactical understanding; and acceptance.

Staff participation in OPM design, planning and ongoing revisions (Element 3.1) was one of the most critical issues cited for successful implementation [30, 34]. Failure in this element is a "common implementation barrier" [14] and dedicated OPM implementation communication (Element 3.2) to show that OPM "never sleeps" [31] can enable staff engagement. Communication reinforces executive commitment [14], and helps embed implementation within business-as-usual activities and addressing any conceptual barriers (Element 3.3).

Encouraging staff learning (Element 3.4) supports improvement in implementation. Engaging stakeholders through periodic and systematic reviews (Element 3.5) builds confidence [33]. OPM champions or change agents (Element 3.6) - especially those with strong internal networks or clinical backgrounds, are valuable in helping people accept implementation initiatives [30]. Development of data analysis skills and feedback learning systems (Element 3.7) is important, as well as team-based inter-disciplinary collaborative approaches (Element 3.8 [31]).

\subsection{Factor 4: Alignment, Integration \& Resourcing}

Aligning organisational strategy and key strategic initiatives (Element 4.1) with OPM implementation was highlighted in 11 of the 20 papers. Radnor and Lovell [29] went so far as to identify a set of eight checklist benefits of OPM strategic alignment and integration, while Inamdar and Kaplan [31] suggested organisations that devote time and resources to OPM implementation have greater success.

Embedding OPM within existing management processes, governance systems, policies and reporting systems with sufficient assigned resources (Element 4.2) is critical [29]. Ensuring measures and indicators are articulated in an easily understood manner (Element 4.3) [14] is necessary, as too. appropriate resourcing investment (Element 4.4) of time, energy and workforce talent [29, 15].

Cascading OPM accountability throughout the organisation (Element 4.5) was identified as a method of integrating OPM into business as usual [14]. Cascading translates high-level strategy down through the organisation into lower level objectives, measures and tactical operational details [28, 27].

\subsection{Factor 5: Measures \& Indicators}

The final factor for OPM implementation success is to ensure organisational performance measures and indicators are developed in a manner that is customised to represent all dimensions of the organisation [14]. OPM should be modified to suit an organisation $[15,7]$. The flexibility to adjust and adapt to individual organisations was identified in 12 of the 20 journal papers and is represented through three operating elements (see Table 3 ).

Developing measures and indicators customised to all of the organisation's dimensions (Element 5.1) is critical. Metrics chosen should relate to the idiosyncrasies and specificities of the sector while also appreciating the organisation's planned processes and targeted outcomes [16, 27, 15]. It is also important to ensure that meaningful objectives or indicators are set for each measure (Element 5.2). Measures and indicators should also match the shared vision amongst the staff cohort (Element 5.3) particularly the clinical and manager cohorts $[27,35]$.

\section{Discussion}

\subsection{The Importance of Sector Specific OPM Approaches}

The lack of a tailored and easy-to-use OPM implementation approach for non-acute health charities may be a factor for its under-utilisation or under-reporting in the sector. The value of tailored implementation OPM processes has been demonstrated in sanitation [5], public services [36], information technology [37], transport [38], training [39] and sports [40]. The NCPI Framework is the first OPM implementation guide to consider the unique characteristics of non-acute health charities and may support OPM uptake by the sector.

While the advantage of OPM implementation tailoring is acknowledged, evidence suggests there are similarities and consistencies in necessary implementation factors across for-profit, government and not-for-profit industry. Nalwoga and van Dijk $[5,62]$ discuss the nine elements of the EFQM OPM model which include leadership, people management, policy and strategy, resources and processes. Ozmantar and Gedikoglu [41] suggest 12 implementation principles in their study of OPM for educational institutions. These include willingness to change; managerial support; appropriate team members; training of staff; distinctive OPM dimensions; smart strategic objectives; open communication system; structured report format. Grey literature also offers generic OPM implementation approaches. One example by Torben Rick [42] recommends six OPM implementation factors using the acronym SIMPLE - Set expectations, invite commitment, measure progress, provide feedback, link to consequences and evaluate effectiveness. While Zimmerman [15] suggests 
successful OPM implementation cannot be assumed by blindly replicating methods from other industry and sectors many of the factors shown above overlap with the NCPI Framework's operating elements and factors. Factors such as connecting to strategy; executive leadership; resourcing; dedicated facilitation staff; organisational specific performance indicators; and monitoring implementation progress, appear to be common threads between the examples studied. This strengthens the likelihood of the NCPI Framework's feasibility and should encourage leaders of non-acute health charities.

\subsection{The Role of Organisational Leaders}

Despite the differences and similarities in OPM implementation between industry types the responsibility of executive leadership in enabling organisational performance cannot be denied [43, 44]. Boards and executive leaders cannot be spectators to OPM. They must turn organisational vision into concrete operations; build cultures that enable learning and change; and report on organisational performance transparently and consistently [44, 43]. The NCPI Framework includes operating elements that require demonstration of Board and executive level commitment, support and direction in each of its five factors.

\subsection{OPM Success Takes More Than a Framework}

As frameworks act as how-to-guides or checklists for implementation [45], the non-acute health charity sector may see the NCPI Framework as a valuable resource as not-for-profit managers often seek easy-to-follow activity lists to bring about organisational change [25]. However, caution is necessary as implementation of organisational initiatives, like OPM, often require multi-layered models to complement tactical frameworks $[46,45]$. Consistent with Organisational Learning Theory $[47,2]$ and action implementation approaches [48, 49], implementation models describe the phenomenon being activated, articulate the phases necessary to embed the phenomenon, and bring to life the relationship between theoretical reasoning and action. They drive the whole-oforganisation experience, culture and learning processes necessary to develop systemic commitment, investment necessary, and renewal in knowledge and behaviour [47, $50,2]$. The authors acknowledge that the NCPI Framework is in essence a tactical activity list and further work may be necessary to develop an OPM implementation model which also takes into account theoretical groundings and guiding principles.

\subsection{Building Capability for Success}

While organisational performance measurement might be a feature of success in today's business environment, paradoxically, it may be the levels of capability necessary to successfully implement OPM that has been a bridge too far for non-acute health charities. Potentially, non-adopters of OPM may not have, or do not expect to have, the necessary capabilities to implement significant organisational development initiatives [51]. Most non-profit funders take a project-based rather than organisation building approaches and authors such as Macmillan, Paine, Kara, Dayson, Sanderson and Wells [52] have called on funders to support more systematic understanding of, and response to, the dynamics of capability building in nonprofit and voluntary sectors. Direct assistance to advance awareness, technical skills, resources, infrastructure and leadership development within the sector may also enable OPM.

\subsection{Further Development}

The authors intend to continue case study research of the NCPI Framework's effectiveness in enabling OPM implementation and the value of its inclusion within an OPM model for the sector. Further exploration is recommended to understand the barriers to undertaking OPM, and the most relevant performance indicators and metrics. The ultimate aim should be to evaluate whether OPM implementation impacts overall organisational performance improvement of non-acute health charities.

\section{Conclusion}

Organisational performance measurement (OPM) is a recognised management tool for business success, however it is under-reported and potentially under-utilised by nonacute health charities. The study achieved its aim by identifying factors for implementation of OPM for the nonacute health charity organisations. The resulting framework, titled Framework for Non-Acute Health Charity Performance Implementation (NCPI Framework) packages five implementation factors incorporating 30 operating elements.

The NCPI Framework has elements common to OPM models developed for other industries, however the literature strongly acknowledges the need to tailoring and nuancing to suit specific sectors and individual organisations. As such, the NCPI Framework is unique and may fill a void and support the uptake of OPM within the sector. Further study to validate the NCPI Framework effectiveness within case study organisations would be valuable, as too investigation into the most relevant performance indicators and metrics. Validating the long-term impact of the full-scaled deployment of a tailored OPM in this sector should be the ultimate aim of future studies.

\section{Ethics Approval}

Ethics approval was not required for this study.

\section{Conflicts of Interest}

The authors declare that they have no competing interests. 


\section{References}

[1] Griseri, P. (2013). An introduction to the Philosophy of Management. Sage Publications. ISBN: 9781446246962.

[2] Jones, G. (2013). Organizational theory, design, and change. Pearson.

[3] Sen, D. \& Vayvay, O. (2017). Strategic Enterprise Management for Innovative Companies: The Last Decade of the Balanced Scorecard. International Journal of Asian Social $\begin{array}{lllll}\text { Science, } & 7 & (1): & 97-109 . & \text { doi: }\end{array}$ 10.18488/journal.1/2017.7.1/1.1.97.109.

[4] Carneiro-da-Cunha, J., Hourneaux, F. \& Correa, H. (2016). Evolution and chronology of the organisational performance measurement field. International Journal of Business Performance Management, 17 (2). doi: 10.1504/IJBPM.2016.075553.

[5] Nalwoga, M. \& van Dijk, M. (2016). Organisational performance measurement models, also for poverty alleviation. Int. J. Water, $10 \quad(2 / 3), \quad 122$ : $138 . \quad$ doi: 10.1504/IJW.2016.075564.

[6] Richard, P., Devinney, T., Yip, S., \& Johnson, G. (2009). Measuring organizational performance: towards methodological best practice. Journal of Management, 35 (3), 718-804. doi: 10.1177/0149206308330560.

[7] Kaplan, R. \& Norton, D. (1992) The Balanced Scorecard: Measures that Drive Performance Harvard Business Review, January-February, 710-79.

[8] Bititci, U. (2015). Managing Business Performance: The Science and the Art. John Wiley \& Sons, Ltd.

[9] Aboramadan, M. \& Borgonovi, E. (2016). Strategic management practices as a key determinant of superior nongovernmental organizational performance. Problems of Management in the $21^{\text {st }}$ Century, 11 (2), 710-92.

[10] Tweedie, D. (2016). Not-for-Profit Accountability: Addressing potential barriers. In Butcher, J. \& Gilchrist, D (Eds), The three sector solution: delivering public policy in collaboration with not-for-profits and business (pp 215-233). ANU Press.

[11] Clancey, G. \& Westcott, H. (2017). "This rabid fight for survival": Small NGO manager's experiences of funding reform. Australian Journal of Social Issues, 52 (2), 163-179. https://doi.org/10.1002/ajs4.9.

[12] Soysa, I., Jayamaha, N., \& Grigg, N. (2016). Operationalising performance measurement dimensions for the Australasian nonprofit healthcare sector. The TQM Journal, (28) 6, 954973. https://doi.org/10.1108/TQM-08-2015-0109.

[13] Hardwick, R., Anderson, R. \& Cooper, C. (2015). How do third sector organisations use research and other knowledge? A systematic scoping review. Implementation Sci, 10, 84 https://doi.org/10.1186/s13012-015-0265-6.

[14] Schalm, C. (2008). Implementing a balanced scorecard as a strategic management tool in a long-term care organization. Journal of Health Services Research \& Policy, 1 (13), 8-14. doi: 10.1258/jhsrp.2007.007013.

[15] Zimmerman, J. (2009). Using a balanced scorecard in a nonprofit organization. Nonprofit World, 27, 10-12.

[16] Boateng, A., Akamavi, R. and Ndoro, G. (2015). Measuring performance of non-profit organisations: evidence from large charities. Business Ethics: A European Review, 25 (1), 59-74.

[17] Dickerson, C., Grills, N., Henwood, N., Jeffreys, S., and Lankester, T. (2012). The World Health Organization Engaging with Civil Society Networks to Promote Primary Health Care: A Case Study. Global Health Governance, VI (1) http://blogs.shu.edu/ghg/files/2012/12/GHGJ-VOLUME-VIISSUE-1-FALL-2012-The-World-Health-OrganizationEngaging-with-Civil-Society-Networks-to-Promote-PrimaryHealth-Care-A-Case-Study.pdf.

[18] Colbran, R., Ramsden, R., Stagnitti, K., \& Toumbourou, J. W. (2019). Advancing towards contemporary practice: a systematic review of organisational performance measures for non-acute health charities. BMC Health Services Research, 19 (132). doi: 10.1186/s12913-019-3952-1.

[19] State Government of Victoria (Department of Health and Human Services). Maintenance Care. https://www2.health.vic.gov.au/hospitals-and-healthservices/patient-care/rehabilitation-complexcare/maintenance-care.

[20] Myburgh, C., Brandborg-Olsen, D., Albert, H. \& Hestbaek, L. (2013). The Nordic maintenance care program: what is maintenance care? Interview based survey of Danish chiropractors. Chiropr Man Therap, 21 (27). https://doi.org/10.1186/2045-709X-21-27.

[21] Cortis, N., Lee, I., Powell, A., Simnett, R., and Reeve, R. (2015) Australian Charities Report 2014. Centre for Social Impact and Social Policy Research Centre, UNSW Australia. https://www.csi.edu.au/media/Australian_Charities_Report_2 015_Web_ND8DU2P.pdf.

[22] Colbran, R., Ramsden, R., Stagnitti, K., \& Adams, S. (2017). Measures to assess the performance of an Australian nongovernment charitable non-acute health service: A Delphi Survey of Organisational Stakeholders. Health Management Services Research Journal, 31 (1), 11-20. https://doi.org/10.1177/0951484817725681.

[23] Hitch, D., Taylor, M., Pepin, G., \& Stagnitti, K. (2012). Evidence based guidelines to improve engagement and participation for people experiencing depression. International journal of psychosocial rehabilitation, 17 (1), 10-22.

[24] Ryan, G. \& Bernard, R. (2003). Techniques to identify themes. Field Methods, 15 (1), 85-109.

[25] Niven, P. R. (2008). Balanced scorecard step-by-step for government and nonprofit agencies. Hoboken, N. J: J. Wiley \& Sons.

[26] Nair M. (2004). Essentials of balanced scorecard. Hoboken, NJ: Wiley.

[27] Bisbe, J. and Barrubés, J. (2012). The Balanced Scorecard as a Management Tool for Assessing and Monitoring Strategy Implementation in Health Care Organizations. Revista Española de Cardiología (English Edition), 65 (10), 919-927.

[28] Sharma, A, 2008, 'Implementing Balance Scorecard for Performance Measurement', The Icfai University Journal of Business Strategy, vol. VI, no. 1, pp: 7-16. 
[29] Radnor, Z, \& Lovell, B, 2003/1, 'Defining, justifying and implementing the Balanced Scorecard in the National Health Service', International Journal of Medical Marketing, vol. 3, no. 3, pp. 174-188.

[30] Kollberg, B, \& Elg, M, 2011, 'The practice of the Balanced Scorecard in health care services' International Journal of Productivity and Performance Management, vol. 60, no. 5, pp: $427-445$.

[31] Inamdar, N., \& Kaplan, R. (2002). Applying the Balanced Scorecard in Healthcare Provider Organizations. Journal of Healthcare Management, 47 (3), 179-195.

[32] Smith, RF, 2007, 'Business process management and the balanced scorecard: Using processes as strategic drivers', Hoboken: John Wiley.

[33] Grigoroudis, E, Orfanoudaki, E, Zopounidis, C, 2012, 'Strategic performance measurement in a healthcare organisation: a multiple criteria approach based on balanced scorecard', Omega, vol. 40, no. 1, pp: 104-19.

[34] Martello, M., Watson, J. G. \& Fisher, M. J. (2018). Implementing A Balanced Scorecard In A Not-For-Profit Organization. Journal of Business \& Economics Research, 6 (9), 67-80.

[35] Groene, O., Brandt, E., Schmidt, W., \& Moeller, J. (2009). The Balanced Scorecard of acute settings: Development process, definition of 20 strategic objectives and implementation. International Journal for Quality in Health Care, 21 (4), 259-271. doi: 10.1093/intqhe/mzp024.

[36] Ancarani, A, 2009, 'Supplier evaluation in local public service: application of a model of value for customer', Journal of Purchasing \& Supply Management, Vol. 15, No. 1, pp. 33-42.

[37] El Yamami, A., Mansouri, K., Qbadou, M., \& Illoussamen, E. H. (2019). Introducing ITIL framework in small enterprises: tailoring ITSM practices to the size of company. International Journal of Information Technologies and Systems Approach (IJITSA), 12 (1), 10-19.

[38] Tubis, A., \& Werbińska-Wojciechowska, S. (2017). Balanced scorecard use in passenger transport companies performing at Polish market. Procedia Engineering, 187 (1), 538-547.

[39] Smolska, M. (2020). The Implementation Process of Balanced Scorecard of a Training Company. Zeszyty Naukowe. Organizacja i Zarządzanie/Politechnika Śląska, (144), 449458 .

[40] Dimitropoulos, P., Kosmas, I., \& Douvis, I. (2017). Implementing the balanced scorecard in a local government sport organization. International Journal of Productivity and Performance Management.

[41] Ozmantar, Z. K., \& Gedikoglu, T. (2016). Design principles for the development of the balanced scorecard. International Journal of Educational Management.

[42] Rick, T, 2014, 'How to ensure successful performance measurement', https://www.torbenrick.eu/t/r/cwo (accessed 21 March 2020)

[43] Odor, H. (2018). A literature review on organisational learning and learning organisations. International Journal of Economics \& Management Sciences, 7 (1), 494. Doi: $10.4172 / 2162-6359.1000494$.
[44] Guta, AL, 2015, 'An analysis of factors that influence organisational learning: The case of higher education institutions', 15th International Academic Conference, Rome. doi: 10.20472/IAC.2015.015.067. https://www.iises.net/proceedings/international-academicconference-rome/table-of-content cid $=10 \&$ iid $=067 \&$ rid $=3387$.

[45] Nilsen, P, 2015, 'Making sense of implementation theories, models and frameworks', Implementation Science, vol. 10. https://implementationscience.biomedcentral.com/articles/a0.1 186/s13012-015-0242-0.

[46] Bergeron, K., Abdi, S., DeCorby, K., Mensah, G., Rempel, B., \& Manson, H. (2017). Theories, models and frameworks used in capacity building interventions relevant to public health: a systematic review. BMC public health, 17 (1), 10-12.

[47] Argote, L. (2013). Organizational learning: creating, retaining and transferring knowledge. Springer.

[48] Graham, I, Logan, J, Harrison, M, Strauss, S, Tetroe, J, Caswell, W, \& Robinson, N, 2006, 'Lost in Knowledge Translation: Time for a Map?' The Journal of Continuing Education in the Health Professions, vol. 26, pp: 13-24.

[49] Greenhalgh, T, Robert, G, MacFarlane, F, Bate, P, \& Kyriakidou, O, 2004, 'Diffusion of Innovations in Service Organizations: Systematic Review and Recommendations' The Milbank Quarterly, vol. 82, no. 4, pp: 5810-629 https://doi.org/a0.1111/j.0887-378X.2004.00325.x https://onlinelibrary.wiley.com/doi/full/a0.1111/j.0887378X.2004.00325.x.

[50] Ricciardi, F., Cantino, V. \& Rossignoli, C. (2020): Organisational learning for the common good: an emerging model. Knowledge Management Research \& Practice, doi: 10.1080/14778238.2019.1673676.

[51] Lueg, R., \& Vu, L. (2015). Success factors in Balanced Scorecard implementations-A literature review. management revue, 306-327.

[52] Macmillan, R., Ellis Paine, A., Kara, H., Dayson, C., Sanderson, E. \& Wells, P. (2014) Building capabilities in the voluntary sector: What the evidence tells us. TSRC Research Report 125, Birmingham: TSRC. https://www.birmingham.ac.uk/Documents/college-socialsciences/social-policy/tsrc/reports/research-report-125building-capabilities.pdf.

[53] Chen, S, 2010, 'The establishment and comparison of the balanced scorecard for profit and non-profit organizations', African Journal of Business Management, vol. 4, no. 14, pp: 3005-3012.

[54] Kong, E, 2010, 'Analysing BSC and IC's usefuleness in nonprofit organisations', Journal of Intellectual Capital, 11 (3). pp. 284-304. ISSN 1469-1930.

[55] Weir, E, d'Entremont, N, Stalker, S, Kurji, K, \& Robinson, V, 2009, 'Applying the balanced scorecard to local public health performance measurement: deliberations and decisions' BMC Public Health, vol. 9, no. 1, doi: 10.1186/1471-2458-9-127.

[56] Gurd, B, \& Gao, T, 2007, 'Lives in the balance: an analysis of the balanced scorecard (BSC) in healthcare organizations, International Journal of Productivity and Performance Management, vol. 57, no. 1, pp: 6-21.

[57] Ronchetti, JL, (2006). An integrated Balanced Scorecard strategic planning model for nonprofit organizations. Journal of practical consulting, vol. 1, no 1, pp: 25-35. 
[58] Yeung, A, \& Connell, J, 2006, 'The Application of Niven's Balanced Scorecard in a Not-For-Profit Organization in Hong Kong: What are the Factors for Success', Journal of Asia Business Studies, vol. 1, no. 1.

[59] Yang, C, Cheng, L, Yang, C, 2005, 'A study of implementing Balanced Scorecard (BSC) in non-profit organizations: A case study of private hospital', Human Systems Management, vol. 24, no. 4, pp. 285-300.
[60] Markham, C, 2003, 'Charity's changed environment', CMA Management, vol. 76, no. 9, pp: 24-28.

[61] Radnor, Z, \& Lovell, B, 2003/2, 'Success factors for implementation of the balanced scorecard in a HNS multiagency setting', International Journal of Health Care Quality Assurance, vol. 16, no. 2, pp: 99-108.

[62] EFQM (1996) Self-Assessment 1996 Guidelines, Brussels, Belgium. 